\title{
Protein complexes in bacterial and yeast mitochondrial membranes differ in their sensitivity towards dissociation by SDS
}

\author{
Jacob Gubbens $^{\mathrm{a}, *}{ }^{\text {, Monique Slijper }}{ }^{\mathrm{b}}$, Ben de Kruijff ${ }^{\mathrm{a}}$, Anton I.P.M. de Kroon ${ }^{\mathrm{a}, 1}$

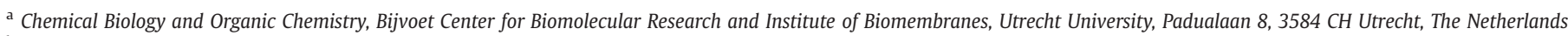

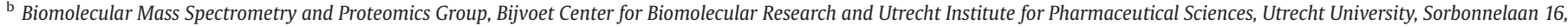 \\ 3584 CA Utrecht, The Netherlands
}

\section{A R T I C L E I N F O}

\section{Article history:}

Received 29 May 2008

Received in revised form 20 August 2008

Accepted 22 August 2008

Available online 11 September 2008

\section{Keywords:}

Yeast

Mitochondria

Membrane protein complex

2D gel electrophoresis

Proteomic

\begin{abstract}
A B S T R A C T
Previously, a 2D gel electrophoresis approach was developed for the Escherichia coli inner membrane, which detects membrane protein complexes that are stable in sodium dodecyl sulfate (SDS) at room temperature, and dissociate under the influence of trifluoroethanol [R. E. Spelbrink et al., J. Biol. Chem. 280 (2005), 28742-8]. Here, the method was applied to the evolutionarily related mitochondrial inner membrane that was isolated from the yeast Saccharomyces cerevisiae. Surprisingly, only very few proteins were found to be dissociated by trifluoroethanol of which $L p d 1 \mathrm{p}$, a component of multiple protein complexes localized in the mitochondrial matrix, is the most prominent. Usage of either milder or more stringent conditions did not yield any additional proteins that were released by fluorinated alcohols. This strongly suggests that membrane protein complexes in yeast are less stable in SDS solution than their E. coli counterparts, which might be due to the overall reduced hydrophobicity of mitochondrial transmembrane proteins.
\end{abstract}

(c) 2008 Elsevier B.V. All rights reserved.

\section{Introduction}

Although up to $30 \%$ of all open reading frames encode membrane proteins [1], they are still highly underrepresented in proteomic and structural studies. This may be caused by low levels of expression and the presence of exposed hydrophobic segments that complicate proper solubilization. Since membrane proteins often function in large oligomeric complexes, blue native (BN)-polyacrylamide gel electrophoresis (PAGE) has been developed to study the membrane proteome at the level of protein complexes by separating them in their enzymatically active form after solubilizing the membrane in nondenaturing detergents [2]. In combination with a second dimension separation by sodium dodecyl sulfate (SDS)-PAGE to dissociate the complexes in their individual subunits, important structural information can be obtained, including subunit composition [3] and stability of (super-)complexes [4].

Recently, an SDS-TFE-SDS-PAGE approach was developed that is complementary to BN-SDS-PAGE in that it detects membrane protein complexes that are stable in SDS at room temperature [5]. SDS-stable

\footnotetext{
* Corresponding author. Tel.: +31 30 2533345; fax: +31 302533969.

E-mail address: j.gubbens@uu.nl (J. Gubbens).

${ }^{1}$ Current address: Membrane Enzymology, Bijvoet Center for Biomolecular Research and Institute of Biomembranes, Utrecht University, Padualaan 8, $3584 \mathrm{CH}$ Utrecht, The Netherlands.
}

complexes were shown to dissociate under the influence of small, fluorinated, alcohols such as 2,2,2-trifluoroethanol (TFE) that are thought to disturb the local interactions between hydrophobic protein segments, and/or between the hydrophobic protein segments and the surrounding membrane lipids or SDS-molecules [5-8]. In addition, TFE has been used at high concentrations $(\geq 50 \%)$ to improve the separation of membrane proteins from E. coli [9] and Streptococcus mutans [10] in the classic 2D iso-electric focusing (IEF)-SDS-PAGE approach, illustrating its potential to solubilize membrane proteins. In contrast, TFE is generally regarded as a stabilizing agent for soluble, globular proteins [8,11].

In SDS-TFE-SDS-PAGE, samples are separated in the first dimension by SDS-PAGE without prior heating. Subsequently, the gel lanes are exposed to $25 \%(\mathrm{v} / \mathrm{v})$ TFE, and then placed on top of the second dimension SDS-PAGE gel. After electrophoresis in the second dimension, complexes stable in SDS but dissociated by the alcohol will appear as off-diagonal spots. When applied to E. coli inner membrane preparations, 23 integral membrane proteins and 16 peripheral membrane proteins were identified in off-diagonal spots, while only 14 soluble proteins were found. Comparison to the results of two BN-SDS-PAGE studies on E. coli $[12,13]$, revealed an overlap of only 9 integral membrane proteins and 11 peripheral proteins, indicating that, particularly for integral membrane proteins, the two techniques are complementary. For two of the proteins identified in the SDS-TFE-SDS-PAGE study, the preprotein translocase SecA and the 
mechanosensitive channel MscS, the oligomeric state and, in the latter case, its dissociation by TFE was confirmed in one dimensional SDS-PAGE gels using immunodetection $[8,14]$.

Based on the results obtained in E. coli inner membranes, we reasoned that also other membranes may contain membrane protein complexes that escape detection by BN-SDS-PAGE because they resist dissociation by SDS. To test this, the SDS-TFE-SDS-PAGE technique was applied to yeast mitochondria. This organelle has been studied extensively using BN-SDS-PAGE [15]. Moreover, due to their origin as a bacterial endosymbiont [16], they are structurally and evolutionarily related to prokaryotic inner membranes. Surprisingly, using yeast mitochondria, only one off-diagonal spot appeared as a result of the treatment with TFE. LC-MS/MS analysis showed that this spot predominantly contained a predicted matrix protein that might be membrane associated in yeast. We conclude that, under the conditions tested, yeast mitochondrial membranes do not contain integral membrane protein complexes that are stable in SDS, but can be dissociated by TFE, in contrast to E. coli inner membranes. This difference may be due to the overall reduced hydrophobicity of mitochondrial transmembrane proteins compared to that of their counterparts from the $E$. coli inner membrane.

\section{Materials and methods}

\subsection{Isolation of membranes}

Sucrose gradient purified mitochondria and inner mitochondrial membrane vesicles from yeast were isolated from the wild type strain

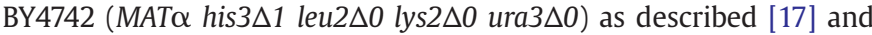
stored in aliquots at $-80{ }^{\circ} \mathrm{C}$ at a protein concentration of approximately $2 \mathrm{mg} / \mathrm{ml}$ in $20 \mathrm{mM}$ HEPES/KOH, $0.5 \mathrm{mM}$ EDTA, $\mathrm{pH}$ 7.4. Inner membrane vesicles from E. coli strain BL21 were purified and stored as described $[5,18]$. Protein concentrations were determined using the BCA method (Pierce, Rockford, IL) with $0.1 \%(w / v)$ SDS added and bovine serum albumin as a standard.

\section{2. $2 \mathrm{D}$ gel electrophoresis}

Aliquots of mitochondrial or E. coli membranes corresponding to either $5 \mu \mathrm{g}$ or $20 \mu \mathrm{g}$ protein, for the mini Protean III or Protean II setup (Biorad, Hercules, CA), respectively, were mixed with SDSPAGE sample buffer (31 mM Tris/HCl, 1.25\% (w/v) SDS, 5.5\% (v/v) glycerol, $25 \mathrm{mM}$ dithiotreitol, $\mathrm{pH} 6.8$ ) at room temperature or at $95{ }^{\circ} \mathrm{C}$ for $5 \mathrm{~min}$. Gel electrophoresis and the incubation of gel strips in $25 \%(\mathrm{v} / \mathrm{v})$ TFE or $25 \%(\mathrm{v} / \mathrm{v})$ 1,1,1,3,3,3-hexafluoroisopropanol (HFIP) in electrophoresis buffer were performed as described [5] with minor modifications. While the first dimension gels were $0.75 \mathrm{~mm}$ thick, the second dimension gels were $1 \mathrm{~mm}$ thick in order to facilitate the transfer of the gel strips on top of the second dimension gels. Before running the second dimension, a filter paper (Whatman, Maidstone, UK) containing 'precision plus' prestained protein marker (Biorad) was placed next to the gel strip and both were embedded in $0.3 \%(\mathrm{w} / \mathrm{v})$ agarose in electrophoresis buffer [19]. After electrophoresis, the gels were washed in $\mathrm{H}_{2} \mathrm{O}$, the mini gels 4 times $5 \mathrm{~min}$ and the large Protean II gels 4 times $10 \mathrm{~min}$, and proteins were stained using the 'blue silver' colloidal Coomassie staining protocol [20].

Where indicated, lithium dodecyl sulfate (LDS)-PAGE instead of SDS-PAGE was used in the first dimension. In this case, SDS in the sample buffer, electrophoresis buffer and gels was replaced by LDS at corresponding concentrations. All handling was performed on ice and the gels were run at a maximum power of $2 \mathrm{~W} /$ gel to prevent heating. The resulting current never exceeded $15 \mathrm{~mA} / \mathrm{gel}$. Incubation of the gel strips and running of the second dimension were performed at room temperature.

\subsection{LC-MS/MS analysis for protein identification}

Spots of interest were excised, in gel digested with trypsin and peptides analyzed by nanoscale LC-MS/MS by coupling an Agilent 1100 Series LC system to a LTQ XL quadrupole ion trap mass spectrometer (Finnigan, San Jose, CA), as described [17]. The only modification was that, after digestion, the peptides were extracted using $5 \%(\mathrm{v} / \mathrm{v})$ formic acid instead of acetic acid. Tandem mass spectra were extracted and charge state deconvoluted by BioWorks (Thermo Scientific, Waltham, MA; version 3.3). All MS/MS samples were analyzed using Mascot (Matrix Science, London, UK; version 2.2.1) and X! Tandem (www.thegpm.org; version 2007.01.01.1), both set up to search the Yeast SGD database (5779 entries) with a parent ion tolerance of $0.5 \mathrm{Da}$ and a fragment ion mass tolerance of $0.9 \mathrm{Da}$. Fixed and variable modifications were the iodoacetamide derivative of cysteine and oxidation of methionine, respectively. Scaffold (version 01_07_00, Proteome Software, Portland, OR) was used to validate MS/ MS based peptide and protein identifications. Peptide identifications were accepted if they could be established at greater than 95.0\% probability as specified by the Peptide Prophet algorithm [21]. Protein identifications were accepted if they could be established at greater than $99.0 \%$ probability as specified by the Protein Prophet algorithm [22], and contained at least 2 identified peptides in one of the samples. Proteins that contained similar peptides and could not be differentiated based on MS/MS analysis alone were grouped to satisfy the principles of parsimony.

\subsection{BLAST analysis}

BLASTP [23,24] (NCBI; version 2.2.17) was used to find yeast homologues of the E. coli proteins identified in a previous study [5]. Each $E$. coli sequence was searched against non-redundant sequences from the Swissprot database, limiting the search results to sequences from S. cerevisiae (taxid 4932) and using default scoring parameters.

\section{Results}

3.1. Analysis of yeast mitochondria by an SDS-TFE-SDS-PAGE approach yields only one TFE-dependent off-diagonal spot

The SDS-TFE-SDS-PAGE approach was applied to E. coli inner membranes (Fig. 1A) and mitochondria from the yeast $S$. cerevisiae (Fig. 1B), using improved methods for transfer of proteins into the second dimension gel and for Coomassie staining. Compared to the previous study in E. coli (Fig. 3 in [5]), the spots in the diagonal were more focused. Previously described off-diagonal spots were reproduced, as indicated by the arrowheads (Fig. 1A), even though the concentration of SDS in the sample buffer was higher, $1.25 \%(w / v) v$ v. $0.4 \%$ in [5]. In stark contrast to the large number of spots in the E. coli sample, only two off-diagonal spots were found in yeast mitochondria (Fig. 1B), one at $60 \mathrm{kDa}$ and a weak spot at $35 \mathrm{kDa}$. In some gels, an additional spot appeared at $50 \mathrm{kDa}$ (data not shown). Using a lower concentration of SDS $(0.4 \%(\mathrm{w} / \mathrm{v}))$ in the sample buffer did not reveal any additional spots (data not shown). Considering that $1.4 \mathrm{~g}$ of SDS binds to one gram of protein $[25,26]$, it was calculated that $0.4 \%(\mathrm{w} / \mathrm{v})$ SDS corresponds to at least a ten-fold excess.

To test whether the off-diagonal spots indeed represent proteins from SDS-stable protein complexes that dissociate under the influence of TFE, we performed experiments in mini gels using varying conditions. The usage of mini gels facilitated rapid screening, since the electrophoresis is faster and requires less protein. Yeast mitochondria (Fig. 2A) and inner mitochondrial membrane vesicles (data not shown) yielded the same off-diagonal spots after TFE-treatment of the first dimension gel, including the additional spot at $50 \mathrm{kDa}$, very close to the diagonal. Therefore, the presence of soluble proteins in the mitochondrial samples did not seem to interfere with the detection of 

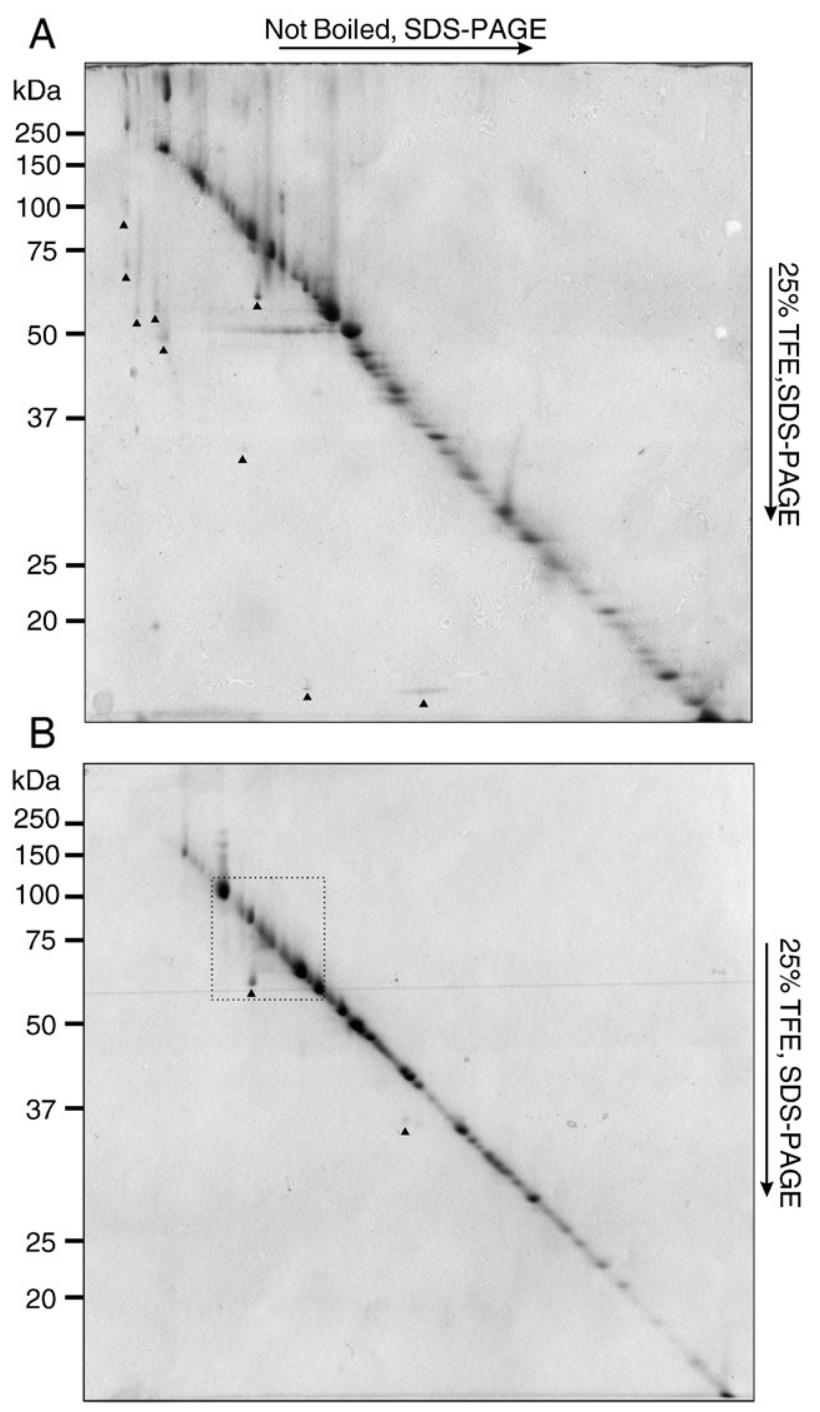

Fig. 1. 2D gels showing dissociation of SDS-stable protein complexes by TFE in E. coli inner membrane vesicles (A) and in yeast mitochondrial membranes (B). The samples were separated on an $18 \mathrm{~cm}$ SDS-PAGE gel, incubated in 25\% (v/v) TFE, and again separated on SDS-PAGE in the second dimension. Proteins were visualized using a colloidal Coomassie stain. The arrowheads indicate the off-diagonal spots described previously (A), and those found in mitochondrial membranes (B). The marked area in panel $\mathrm{B}$ corresponds to the part of the gel shown in Fig. 4B.

off-diagonal spots. Next, the mitochondrial sample was heated in SDSPAGE sample buffer to make sure all protein complexes were dissociated, and subsequently separated on SDS-TFE-SDS-PAGE. Under these conditions, the two off-diagonal spots at $50 \mathrm{kDa}$ and $35 \mathrm{kDa}$ were again observed (Fig. 2B), suggesting that these represent proteins that undergo a conformational change or modification upon exposure to TFE. However, as these spots also appeared after treatment of the gel strip in the absence of any alcohol (see Fig. 3A), they were considered an artifact of the method, possibly caused by the transfer of proteins into the second dimension gel via a stacking gel. The spot at $60 \mathrm{kDa}$, indicated by the large arrowhead in Fig. 2A, did not appear after heating or mock-treatment, confirming that this spot represents one or more mitochondrial proteins present in an SDSstable complex that can be dissociated by TFE.

\subsection{Mitochondrial protein complex stability examined by SDS-HFIP-SDS- PAGE and LDS-TFE-SDS-PAGE}

Based on the appearance of a single TFE-dependent off-diagonal spot, we speculated that mitochondrial protein complexes are either more stable than their counterparts from E. coli, rendering $25 \%(\mathrm{v} / \mathrm{v})$ TFE insufficient for complete dissociation, or less stable, causing them to already completely dissociate in SDS at room temperature. To test the first possibility, HFIP, which has been shown to dissociate the same protein complexes as TFE at a lower concentration [5,7], was used as the most potent alcohol to dissociate SDS-stable protein complexes. Treatment with 25\% (v/v) HFIP yielded one new additional spot (white arrowhead, Fig. 3B) slightly above the $60 \mathrm{kDa}$ TFE-dependent spot (large black arrowhead). This spot at $70 \mathrm{kDa}$ could represent additional proteins dissociated by HFIP. Alternatively, the presence of this spot could be due to smearing caused by HFIP in the second dimension, since additional smearing due to HFIP is observed above the diagonal when Fig. 3B is compared to Fig. 3A (no alcohol) and Fig. 2A (TFE).

In case the protein complexes already dissociate in SDS at room temperature, lowering the temperature might yield extra off-diagonal spots. This was achieved by replacing SDS with LDS, which does not precipitate at low temperatures, thus allowing the sample preparation and electrophoresis to be performed on ice. This method has been successfully applied to study the effect of TFE on the dissociation of $E$. coli MscS (mechanosensitive channel of small conductance), a heptameric complex of identical subunits that was stable in LDS-PAGE on ice, and could be dissociated by adding TFE [8]. When yeast mitochondria were separated by LDS-PAGE on ice and the resulting gel lanes were incubated with or without TFE, no additional off-diagonal spots were detected in the second dimension gel (compare Figs. $3 \mathrm{C}$ and $\mathrm{D}$ to Fig. $2 \mathrm{~A}$ ). We conclude that almost all protein complexes in the mitochondrial membrane dissociate in SDS at room temperature or LDS at reduced temperatures.
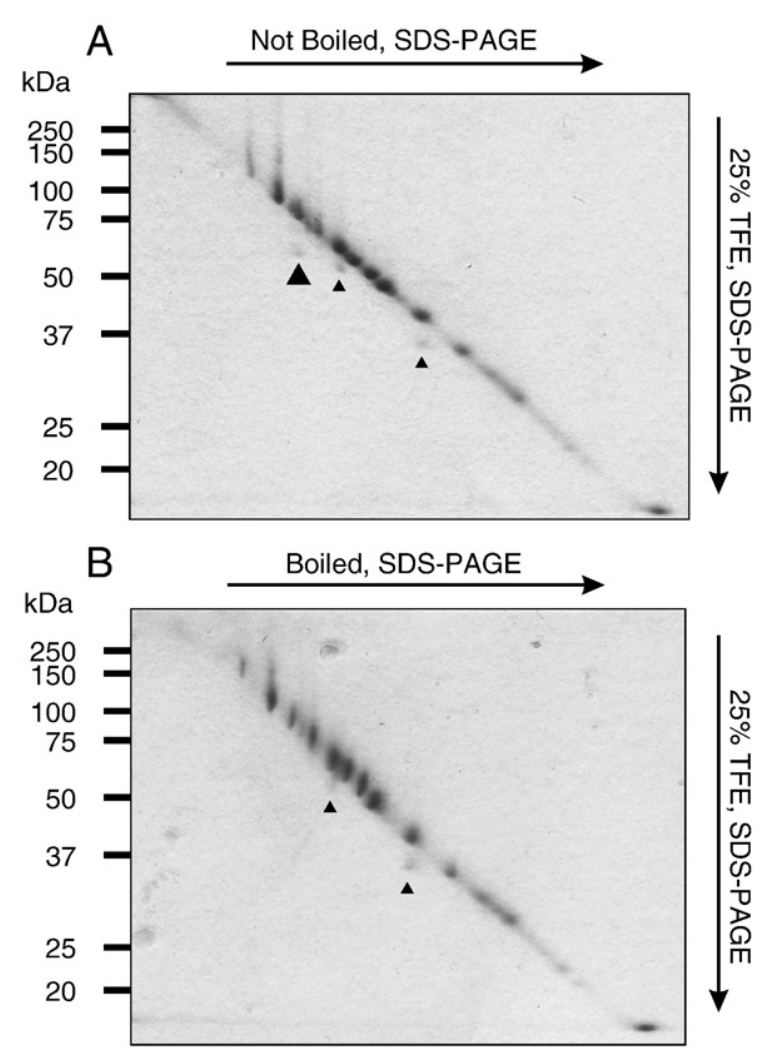

Fig. 2. Dissociation of protein complexes from S. cerevisiae mitochondria by TFE. Yeast mitochondrial proteins were dissolved in SDS-PAGE sample buffer at either room temperature (A) or at $95^{\circ} \mathrm{C}$ for $5 \mathrm{~min}$ (B) and separated on a mini SDS-PAGE gel. After incubation in $25 \%(\mathrm{v} / \mathrm{v})$ TFE, the proteins in the gel lanes were separated on SDS-PAGE in the second dimension. Proteins were visualized using a colloidal Coomassie stain. Arrowheads indicate off-diagonal spots; the large arrowhead indicates the spot only visible in panel A. 
A

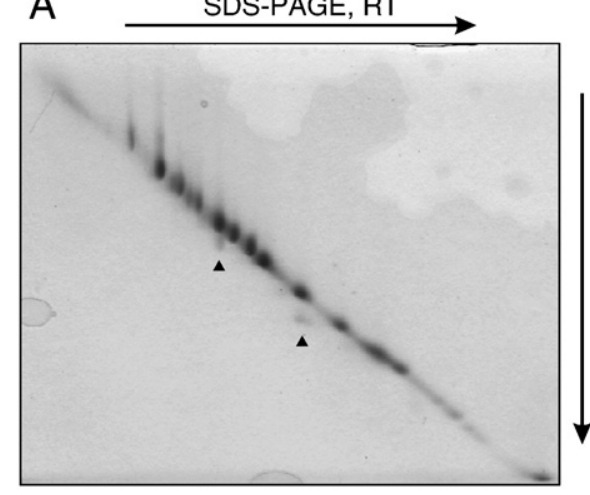

C

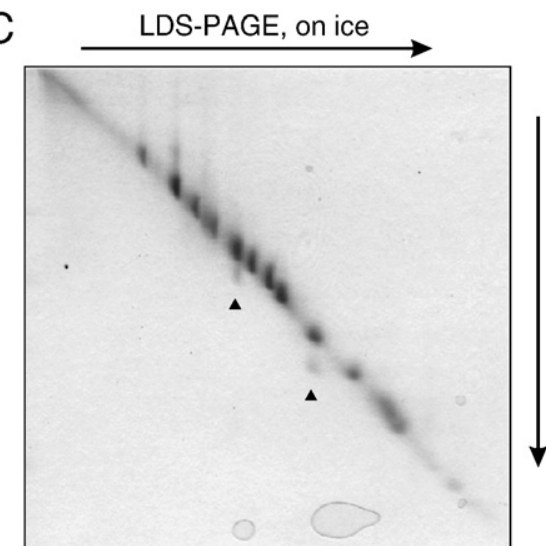

B

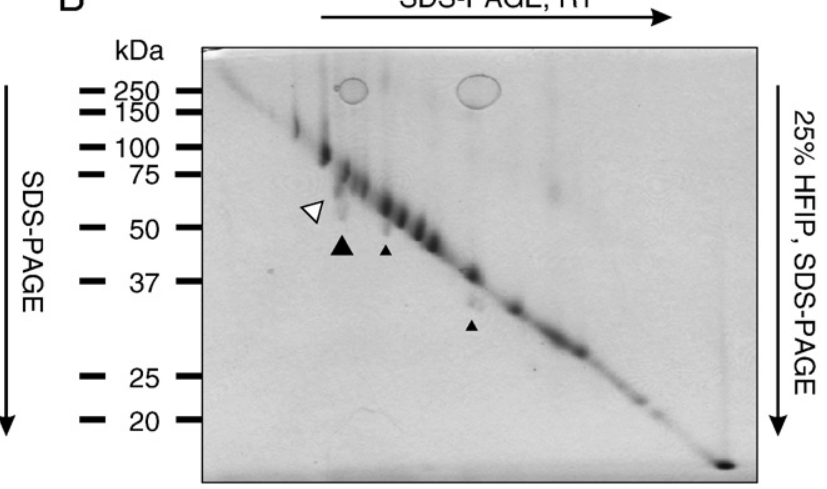

D

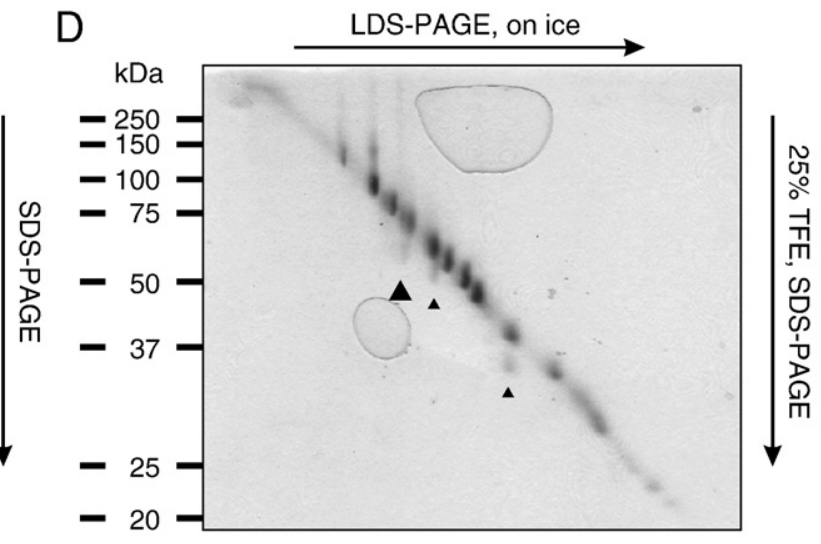

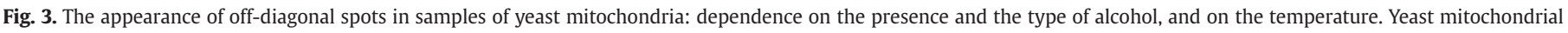

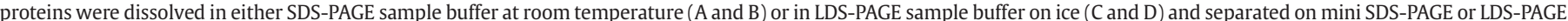

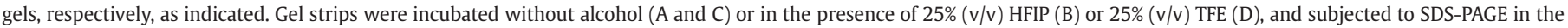

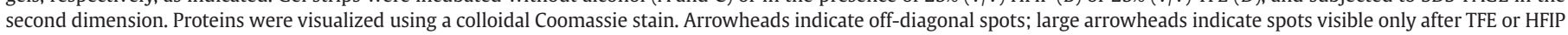
treatment (black), or HFIP treatment only (white).

\subsection{LC-MS/MS analysis of off-diagonal spots}

To identify the proteins migrating in the off-diagonal spots that appear after TFE or HFIP treatment, large 2D gels were prepared after treating the first dimension gel strips with either TFE (Figs. 1B and 4), HFIP, or no alcohol (Fig. 4). Spots 1 (60 kDa) and 2 (70 kDa) were excised from the gels shown in Fig. 4, digested using trypsin, and the proteins present identified by LC-MS/MS analysis. Table 1 lists the number of unique peptides found per protein in each spot from the different gels. A complete listing of all identified proteins and peptides

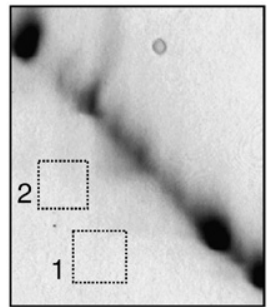

No alcohol

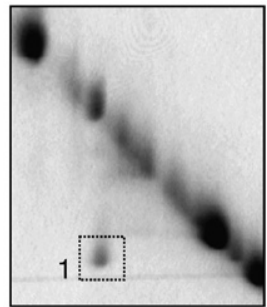

$25 \%$ TFE

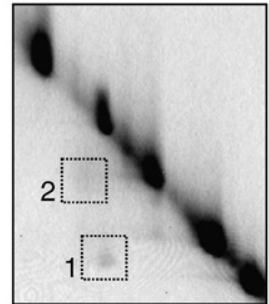

$25 \%$ HFIP
Fig. 4. Dissociation of protein complexes in yeast mitochondria in large gels for use in LC-MS/MS analysis. Yeast mitochondrial proteins were dissolved in SDS-PAGE sample buffer at room temperature and separated on an $18 \mathrm{~cm}$ SDS-PAGE gel. The gel lanes were either not incubated with alcohol, or with $25 \%(\mathrm{v} / \mathrm{v})$ TFE or $25 \%(\mathrm{v} / \mathrm{v})$ HFIP, as indicated. Proteins in the gel lanes were subjected to SDS-PAGE in the second dimension and visualized using a colloidal Coomassie stain. Only the area containing the excised spots 1 and 2 is shown, which corresponds to the marked area in Fig. 1B. can be found in Supplementary Tables S1 and S2, respectively, of the supplementary information. Multiple proteins were detected in the off-diagonal spots, even when no alcohol was used, indicating that in all cases some smearing of proteins occurs in the vicinity of the diagonal. Most identified proteins have a molecular weight in the expected range of 60-70 kDa. Proteins with a molecular weight outside this range were identified with only a low number of peptides.

Nevertheless, when the TFE-treated sample was compared to the untreated sample, one protein, dihydrolipoamide dehydrogenase (Lpd1p), stood out, showing a reproducible and significant increase in the number of unique peptides ( $55 v s$. 25, Table 1 ) and total number of identified spectra (179 vs. 31, Supplementary Table S1) in spot 1 . The number of unique peptide identifications is known to give a rough estimate of protein abundance [27]. The 55 peptides identified after TFE treatment correspond to $91 \%$ sequence coverage. The identification of Lpd1p at the position of spot 1 in the absence of any treatment with alcohol indicates that also under these conditions the protein runs off-diagonal to some extent. Upon HFIP treatment, the number of unique peptides from this protein showed substantial increases in spot 1 as well as in spot 2, indicating that HFIP causes Lpd1p to distribute between these two off-diagonal spots.

Several other proteins, which are indicated in bold in Table 1, showed an increase in the number of unique peptides in spot 1 or spot 2 after treatment with HFIP. Among these, Atp1p, Atp2p [3], and Ndi1p [28] were found previously in a BN-SDS-PAGE or colorless native $(\mathrm{CN})$ SDS-PAGE approach, migrating at their unassociated molecular weight in the second dimension. This implies that they are dissociated from their respective complexes by SDS at room temperature and that their appearance in off-diagonal spots after HFIP treatment in the present 
Table 1

LC-MS/MS analysis of the off-diagonal spots 1 and 2 from Fig. 4

\begin{tabular}{|c|c|c|c|c|c|c|c|c|c|}
\hline \multirow[t]{3}{*}{ Protein } & \multirow[t]{3}{*}{ ORF name } & \multirow{3}{*}{$\begin{array}{l}\text { MW } \\
\text { (kDa) }\end{array}$} & \multicolumn{5}{|c|}{ Number of unique peptides* } & \multirow[t]{3}{*}{$\mathrm{TM}^{\dagger}$} & \multirow[t]{3}{*}{ Localization } \\
\hline & & & \multicolumn{3}{|l|}{ Spot 1} & \multicolumn{2}{|l|}{ Spot 2} & & \\
\hline & & & Mock & TFE & HFIP & Mock & HFIP & & \\
\hline Ach1p & YBL015W & 59 & 11 & 1 & 13 & 23 & 28 & 0 & M, cytosol \\
\hline Aco1p & YLR304C & 85 & 8 & 2 & 3 & 5 & 4 & 0 & matrix \\
\hline Ald4p & YOR374W & 57 & 14 & 1 & 17 & 4 & 9 & 0 & M \\
\hline Atp1p & YBL099W & 59 & 11 & 6 & 14 & 3 & 10 & 0 & MIM \\
\hline Atp2p & YJR121W & 55 & 0 & 0 & 2 & $\mathbf{0}$ & 3 & 0 & MIM \\
\hline Atp4p & YPL078C & 27 & 0 & 0 & 0 & 1 & 2 & 0 & MIM \\
\hline Cat2p & YML042W & 77 & 0 & 0 & 0 & O & 5 & 0 & $\mathrm{M}, \mathrm{PX}$ \\
\hline Cyb2p & YML054C & 66 & 1 & 0 & 0 & 2 & 2 & 0 & MIS \\
\hline Dld1p & YDL174C & 65 & 0 & 0 & 0 & 2 & 4 & 0 & MIM \\
\hline Fmp29p & YER080W & 72 & 1 & 0 & 0 & 2 & 0 & 0 & M \\
\hline Gut2p & YIL155C & 72 & 1 & 0 & 0 & 12 & 14 & 0 & M \\
\hline Hsp60p & YLR259C & 61 & 0 & 0 & 0 & 19 & 26 & 0 & matrix \\
\hline Ilv2p & YMR108W & 75 & 0 & 0 & 0 & 0 & 3 & 0 & $\mathrm{M}$ \\
\hline Ilv3p & YJR016C & 63 & 1 & 0 & 0 & 1 & 5 & 0 & M \\
\hline Ilv5p & YLR355C & 44 & 0 & 0 & 0 & 2 & 1 & 0 & $\mathrm{M}$ \\
\hline Kgd1p & YIL125W & 114 & 3 & 2 & 0 & 1 & 0 & 0 & matrix \\
\hline Leu4p & YNL104C & 68 & 1 & 0 & 0 & 7 & 15 & 0 & $\mathrm{M}, \mathrm{CP}$ \\
\hline Lpd1p & YFL018C & 54 & 25 & 55 & 37 & 5 & 21 & 0 & matrix \\
\hline Nde1p & YMR145C & 63 & 1 & 0 & 2 & 1 & 1 & 0 & M \\
\hline Ndi1p & YML120C & 57 & $\mathbf{0}$ & 0 & 5 & 0 & 0 & 1 & matrix \\
\hline Pet9p & YBL030C & 34 & 0 & 0 & 3 & 5 & 6 & 3 & MIM \\
\hline Pma1p & YGL008C & 100 & 14 & 1 & 0 & 4 & 1 & 10 & PM, M \\
\hline Por1p & YNL055C & 30 & 0 & 0 & 1 & 2 & 5 & 0 & MOM \\
\hline Sdh1p & YKL148C & 70 & 3 & 1 & 0 & 6 & 9 & 0 & MIM \\
\hline Shm1p & YBR263W & 54 & 3 & 0 & 0 & 0 & 0 & 0 & M \\
\hline Ssc1p & YJR045C & 71 & 26 & 14 & 14 & 4 & 5 & 0 & MIM \\
\hline Tom70p & YNL121C & 70 & 5 & 0 & 0 & 1 & 1 & 1 & MOM \\
\hline Ykr016wp & YKR016W & 61 & 2 & 0 & 2 & 0 & 0 & 1 & $\mathrm{M}$ \\
\hline Yor356wp & YOR356W & 70 & 0 & 0 & 0 & 0 & 2 & 0 & M \\
\hline
\end{tabular}

Proteins that show a significant change upon TFE or HFIP treatment are bold-faced and described in the main text.

* Identified in the gel spot after treatment of the sample with no alcohol, 25\% (v/v) TFE or $25 \%(v / v)$ HFIP.

+ Number of transmembrane segments, predicted by the TMHMM algorithm as listed in the Saccharomyces genome database (SGD).

$*$ According to SGD, M = mitochondrial, MIM = mitochondrial inner membrane, MIS = mitochondrial intermembrane space, $\mathrm{MOM}=$ mitochondrial outer membrane, $\mathrm{CP}=$ cytoplasm, PX = peroxisome, $\mathrm{PM}=$ plasma membrane.

study was probably due to smearing. We therefore conclude that Lpd1p is the main protein in both off-diagonal spots 1 and 2.

\section{Discussion}

This study reports a striking difference in the SDS-TFE-SDS-PAGE separation of proteins from $E$. coli inner membranes on the one hand and from yeast mitochondria on the other. Whereas $39 \mathrm{E}$. coli inner membrane proteins were found in off-diagonal spots [5], indicating that they were part of complexes (partly) stable in SDS at room temperature and only dissociated after heating (data not shown) or exposure to TFE, in yeast mitochondria only one spot was found to migrate off-diagonal as a result of TFE treatment. This spot predominantly contained the protein Lpd1p, a flavin-containing component of enzyme complexes present in the mitochondrial matrix, most notably pyruvate dehydrogenase and 2-oxoglutarate dehydrogenase, two homologous complexes that function in the tricarboxylic acid cycle (TCA) cycle. While in bacteria these enzyme complexes are recovered in the soluble cytoplasmic fraction, they were found to be associated with the mitochondrial inner membrane in plants [29] and mammals [30], requiring detergents for solubilization. It was speculated that this association might occur through complex I [29]. Since yeast mitochondria do not contain complex I, the membrane association of Lpd1p remains uncertain. Recently, we found that Lpd1p could be cross-linked to a photoactivatable lipid analogue incorporated in yeast inner mitochondrial membranes
(Gubbens J, Ruijter E, de Fays LEV, Damen JMA, de Kruijff B, Slijper M, Rijkers DTS, Liskamp RMJ, de Kroon AIPM, unpublished results), which indicates a peripheral membrane localization.

It is unclear why Lpd1p ends up in an off-diagonal spot after treatment with TFE or HFIP. It is positioned approximately $20 \mathrm{kDa}$ below the diagonal, but so far no interaction partner of Lpd1p of that size has been discovered. An alternative explanation could be a dramatic influence of fluorinated alcohols on the fold of the protein. Since Lpd1p reportedly is stable at temperatures up to $70{ }^{\circ} \mathrm{C}$ [31], it might not properly unfold in SDS at room temperature, adopting a structure migrating at a higher apparent molecular weight. Upon exposure to TFE or HFIP, this intermediate state may be lost or collapse and as a result an off-diagonal spot is obtained. This is in agreement with the observation that TFE can have a stabilizing effect on the structure of globular proteins and affects the folding of such proteins $[8,11]$. In this scenario, there would be no yeast mitochondrial protein complexes left in SDS for dissociation by fluorinated alcohols.

Table 2

BLAST search for yeast homologues of TFE-released proteins from E. coli

\begin{tabular}{|c|c|c|c|c|c|c|c|}
\hline $\begin{array}{l}\text { E. coli } \\
\text { protein }\end{array}$ & $\begin{array}{l}\text { Swissprot } \\
\text { ID }\end{array}$ & $\begin{array}{l}\text { YeastORF } \\
\text { name }\end{array}$ & Protein & $\begin{array}{l}\text { Score } \\
\text { (bits) }\end{array}$ & $\begin{array}{l}\text { TM* } \\
\text { (GRAVY) }\end{array}$ & $\begin{array}{l}\text { MW } \\
(\mathrm{kDa})\end{array}$ & Localization \\
\hline \multicolumn{8}{|l|}{ Integral } \\
\hline \multirow{6}{*}{$\begin{array}{l}\text { CydD } \\
\text { (GRAVY: } \\
0.154 \text { ) }\end{array}$} & \multirow[t]{6}{*}{ Q8X5I1 } & YMR301C & Atm1p & 148 & $5(0.005)$ & 77.5 & MIM \\
\hline & & YKL209C & Ste6p & 130 & $10(0.078)$ & 144.8 & PM \\
\hline & & YLR188W & Mdl1p & 114 & $5(0.007)$ & 75.9 & MIM \\
\hline & & YPL270W & Mdl2p & 114 & $3(-0.088)$ & 85.1 & MIM \\
\hline & & YLL048C & Ybt1p & 98.2 & $14(0.017)$ & 189.2 & Vacuole \\
\hline & & YDR135C & Ycf1p & 94.7 & $14(0.064)$ & 171.1 & Vacuole \\
\hline \multirow{4}{*}{$\begin{array}{l}\text { HflB } \\
\text { (GRAVY: } \\
-0.259)\end{array}$} & \multirow[t]{4}{*}{ Q8X9L0 } & YPR024W & Yme1p & 450 & $0(-0.355)$ & 81.7 & MIM \\
\hline & & YMR089C & Yta12p & 432 & $0(-0.571)$ & 93.3 & MIM \\
\hline & & YER017C & $\operatorname{Afg} 3 p$ & 430 & $1(-0.363)$ & 84.5 & MIM \\
\hline & & & 17 proteins & $109-216$ & & & \\
\hline \multicolumn{8}{|l|}{ Peripheral } \\
\hline \multirow[t]{3}{*}{ AtpA } & \multirow[t]{3}{*}{ P00822 } & YBL099W & Atp1p & 528 & 0 & 58.6 & MIM \\
\hline & & YJR121W & Atp2p & 114 & 0 & 54.8 & MIM \\
\hline & & YBR127C & Vma2p & 113 & 0 & 57.7 & Vacuole \\
\hline \multirow[t]{3}{*}{ AtpD } & \multirow[t]{3}{*}{ P00824 } & YJR121W & Atp2p & 603 & 0 & 54.8 & MIM \\
\hline & & YBR127C & Vma2p & 108 & 0 & 57.7 & Vacuole \\
\hline & & YBL099W & Atp1p & 98.2 & 0 & 58.6 & MIM \\
\hline LldD & Q8XDF7 & YML054C & Cyb2p & 160 & 0 & 65.5 & MIS \\
\hline \multirow[t]{3}{*}{ FrdA } & \multirow[t]{3}{*}{ Q8XDQ0 } & YKL148C & Sdh1p & 376 & 0 & 70.2 & MIM \\
\hline & & YJL045W & Yjl045wp & 375 & 0 & 69.4 & $\mathrm{M}$ \\
\hline & & YEL047C & Yel047cp & 91.3 & 0 & 50.8 & - \\
\hline GlpD & Q8X6Y5 & YIL155C & Gut2p & 158 & 0 & 72.4 & M \\
\hline \multirow[t]{4}{*}{ LepA } & \multirow{2}{*}{ P60787 } & YLR289W & Guf1p & 528 & 0 & 73.2 & M \\
\hline & & $\begin{array}{l}\text { YOR133W } \\
\text { YDR385W }\end{array}$ & Eft1p/Eft2p & 111 & 0 & 93.3 & Ribosome \\
\hline & P25039 & YLR069C & Mef1p & 108 & 0 & 84.6 & M \\
\hline & P39677 & YJL102W & Mef2p & 92.8 & 0 & 91.3 & M \\
\hline \multicolumn{8}{|l|}{ Unknown } \\
\hline SucA & P07015 & YIL125W & $\operatorname{Kgd} 1 \mathrm{p}$ & 669 & 0 & 114.4 & MATRIX \\
\hline \multirow[t]{2}{*}{ SucB } & \multirow[t]{2}{*}{ P07016 } & YDR148C & $\operatorname{Kgd} 2 \mathrm{p}$ & 301 & 0 & 50.4 & MATRIX \\
\hline & & YNL071W & Lat1p & 146 & 0 & 51.8 & M \\
\hline \multirow[t]{2}{*}{ AceF } & \multirow[t]{2}{*}{ Q8X966 } & YDR148C & $\operatorname{Kgd} 2 p$ & 143 & 0 & 50.4 & MATRIX \\
\hline & & YNL071W & Lat1p & 120 & 0 & 51.8 & M \\
\hline MalP & Q8X708 & YPR160W & Gph1p & 596 & 0 & 103.3 & Cytoplasm \\
\hline SrmB & Q8XA21 & & 23 proteins & $100-226$ & & & \\
\hline YdiJ & Q8X5Y8 & YDL174C & Dld1p & 91.3 & 0 & 65.3 & MIM \\
\hline
\end{tabular}

S. cerevisiae homologues of $E$. coli proteins found in off-diagonal spots after treatment with TFE [5] were searched using BLASTP. Only proteins yielding yeast homologues with a bits score higher than 90 are shown, listed in the same order and using the same classifications as in [5]. E. coli proteins of which yeast homologues were identified in the present study are bold-faced.

* Number of transmembrane domains, predicted by the TMHMM algorithm as listed in the SGD database. Yme1p, Yta12p, and Dld1p are established integral membrane proteins [32], although no transmembrane segment is predicted by TMHMM. For E. coli integral membrane proteins and their homologues, the grand average of hydrophobicity index (GRAVY) is shown in parentheses.

$\dagger$ According to SGD, $\mathrm{M}$ = mitochondrial, $\mathrm{MIM}=$ mitochondrial inner membrane, $\mathrm{MIS}=$ mitochondrial intermembrane space, $\mathrm{PM}=$ plasma membrane.

$\ddagger$ A full list of homologues of HflB and SrmB can be found in the supplementary material, Table S3. 
Apart from Lpd1p, no other mitochondrial protein shows a significant increase in the number of unique peptides in an offdiagonal spot after TFE treatment. Treatment with the more perturbing agent HFIP did not yield any additional proteins migrating in offdiagonal spots. Therefore, the most plausible explanation for our results is that, under the conditions used, all protein complexes in yeast mitochondria are, in contrast to $E$. coli inner membranes, already dissociated by SDS at room temperature (or LDS at $0{ }^{\circ} \mathrm{C}$ ), and cannot be further dissociated, with the possible exception of a Lpd1p-containing complex.

To get insight into the reasons for this striking difference in stability of membrane protein complexes in bacteria and mitochondria, we compared the results obtained in E. coli [5] to our results by performing a BLAST search for yeast homologues of proteins detected in off-diagonal spots in E. coli (Table 2). Most of the yeast homologues found were mitochondrial proteins, in agreement with the evolutionary relationship between mitochondria and prokaryotes. Of the 23 integral inner membrane proteins found in off-diagonal spots in $E$. coli, only two, CydD (Q8X5I1) and HflB / FtsH (Q8X9L0), were found to have homologues in yeast that are considered integral membrane proteins, but these were not identified in the present study. In contrast, 6 out of the 16 peripheral membrane proteins found in E. coli do have yeast homologues, 5 of which were retrieved in off-diagonal spots (Table 2). However, the number of peptides detected for these proteins only increased after HFIP treatment, probably due to smearing as described in the results section. Another protein of which the number of peptides only increased after treatment with HFIP, Dld1p, is a confirmed integral membrane protein [32,33], but is less hydrophobic than its E.coli homologues, whose membrane association is unknown. Interestingly, the $E$. coli homologue of Lpd1p, DldH (P0A9P0, bits score 308), was not found in an offdiagonal spot [5], in contrast to other components of the 2oxoglutarate dehydrogenase complex, SucA (P07015) and SucB (P07016), and of the pyruvate dehydrogenase complex, AceE (P06958) and AceF (Q8X966). We conclude that we were not able to find yeast homologues of $E$. coli membrane proteins in complexes that are stable in SDS and that migrate off-diagonally upon TFE treatment.

In $E$. coli there was only a limited overlap of 9 integral membrane proteins between the datasets obtained after SDS-TFE-SDS-PAGE and BN-SDS-PAGE separation [5,12,13], indicating that in this organism the approaches are complementary. In yeast mitochondria, only one TFEsensitive protein not integral to the membrane could be identified using our approach. We propose that this is related to the fact that the cytosolically synthesized mitochondrial proteins need to be imported into the organelle. The mitochondrial import machinery imposes limits on the overall hydrophobicity of membrane proteins, and on the hydrophobicity of individual transmembrane segments [34], and it also requires the presence of prolines in one or more transmembrane segments of most proteins spanning the inner membrane multiple times [32]. These limitations imply that transmembrane segments of mitochondrial membrane proteins have different properties than those of proteins in other membranes, as illustrated by the failure of the hidden Markov model based TMHMM algorithm [35] to properly predict the transmembrane segments of the established integral inner membrane proteins Dld1p (Tables 1 and 2), Yme1p and Yta12p (Table 2) $[32,33,36]$. Moreover, the grand average of hydrophobicity (GRAVY) [37] is consistently lower for the yeast homologues of the $E$. coli integral membrane proteins in Table 2, with mitochondrial inner membrane proteins yielding the lowest values. In particular, the reduced hydrophobicity of mitochondrial membrane proteins would explain why all protein complexes in this organelle are already dissociated in SDS at room temperature.

In contrast, most mitochondrially encoded proteins are highly hydrophobic membrane proteins [34], raising the question why these were not detected in off-diagonal spots by the SDS-TFE-SDS-PAGE approach. One of these proteins, Oli1p or subunit 9 of the $F_{0}$ part of the
$\mathrm{F}_{1} \mathrm{~F}_{0}$ ATP-synthase, forms a ring consisting of twelve identical subunits and has been found in an oligomeric form after BN-SDS-PAGE separation [3]. Moreover this protein has been found in SDS-resistant complexes together with Atp6p [38] or Oxa1p [39]. Both complexes could be dissociated by TCA precipitation. Oli1p was not found in an offdiagonal spot in the present study, possibly due to its small size of less than $8 \mathrm{kDa}$, causing it to migrate off the gel in the second dimension.

We conclude that, in contrast to E. coli inner membranes, we could not detect protein complexes intrinsic to yeast mitochondrial membranes that are stable in SDS but can be dissociated by fluorinated alcohols such as TFE or HFIP. Therefore, this research demonstrates a surprising difference in membrane protein complex stability of different, but structurally related, biological membranes.

\section{Acknowledgments}

We thank Robin Spelbrink for providing inner membrane vesicles isolated from E. coli, Pieter Rijken for performing pilot experiments, and Mirjam Damen for her help with the LC-MS/MS analysis. This work was supported by the Netherlands Proteomics Centre.

\section{Appendix A. Supplementary data}

Supplementary data associated with this article can be found, in the online version, at doi:10.1016/j.bbapap.2008.08.020.

\section{References}

[1] E. Wallin, G. von Heijne, Genome-wide analysis of integral membrane proteins from eubacterial, archaean, and eukaryotic organisms, Protein Sci. 7 (1998) 1029-1038.

[2] H. Schagger, G. von Jagow, Blue native electrophoresis for isolation of membrane protein complexes in enzymatically active form, Anal. Biochem. 199 (1991) 223-231.

[3] I. Arnold, K. Pfeiffer, W. Neupert, R.A. Stuart, H. Schagger, Yeast mitochondrial F1F0-ATP synthase exists as a dimer: identification of three dimer-specific subunits, EMBO J. 17 (1998) 7170-7178.

[4] K. Pfeiffer, V. Gohil, R.A. Stuart, C. Hunte, U. Brandt, M.L. Greenberg, H. Schagger, Cardiolipin stabilizes respiratory chain supercomplexes, J. Biol. Chem. 278 (2003) 52873-52880.

[5] R.E. Spelbrink, A. Kolkman, M. Slijper, J.A. Killian, B. de Kruijff, Detection and identification of stable oligomeric protein complexes in Escherichi coli inner membranes: a proteomics approach, J. Biol. Chem. 280 (2005) 28742-28748.

[6] E. van den Brink-van der Laan, V. Chupin, J.A. Killian, B. de Kruijff, Stability of KcsA tetramer depends on membrane lateral pressure, Biochemistry 43 (2004) 4240-4250.

[7] E. van den Brink-van der Laan, V. Chupin, J.A. Killian, B. de Kruijff, Small alcohols destabilize the KcsA tetramer via their effect on the membrane lateral pressure, Biochemistry 43 (2004) 5937-5942.

[8] B. Akitake, R.E. Spelbrink, A. Anishkin, J.A. Killian, B de Kruijff, S. Sukharev, 2,2,2Trifluoroethanol changes the transition kinetics and subunit interactions in the small bacterial mechanosensitive channel MscS, Biophys. J. 92 (2007) 2771-2784.

[9] J.M. Deshusses, J.A. Burgess, A. Scherl, Y. Wenger, N. Walter, V. Converset, S. Paesano, G.L. Corthals, D.F. Hochstrasser, J.C. Sanchez, Exploitation of specific properties of trifluoroethanol for extraction and separation of membrane proteins, Proteomics 3 (2003) 1418-1424.

[10] K. Zuobi-Hasona, P.J. Crowley, A. Hasona, A.S. Bleiweis, L.J. Brady, Solubilization of cellular membrane proteins from Streptococcus mutans for two-dimensional gel electrophoresis, Electrophoresis 26 (2005) 1200-1205

[11] M. Buck, Trifluoroethanol and colleagues: cosolvents come of age. Recent studies with peptides and proteins, Q. Rev. Biophys. 31 (1998) 297-355.

[12] F. Stenberg, P. Chovanec, S.L. Maslen, C.V. Robinson, L.L. Ilag, G. von Heijne, D.O. Daley, Protein complexes of the Escherichia coli cell envelope, J. Biol. Chem. 280 (2005) 34409-34419.

[13] J.P. Lasserre, E. Beyne, S. Pyndiah, D. Lapaillerie, S. Claverol, M. Bonneu, A complexomic study of Escherichia coli using two-dimensional blue native/SDS polyacrylamide gel electrophoresis, Electrophoresis 27 (2006) 3306-3321.

[14] J. de Keyzer, E.O. van der Sluis, R.E. Spelbrink, N. Nijstad, B. de Kruijff, N. Nouwen, C. van der Does, A.J. Driessen, Covalently dimerized SecA is functional in protein translocation, J. Biol. Chem. 280 (2005) 35255-35260.

[15] F. Krause, Detection and analysis of protein-protein interactions in organellar and prokaryotic proteomes by native gel electrophoresis: (membrane) protein complexes and supercomplexes, Electrophoresis 27 (2006) 2759-2781.

[16] M.W. Gray, G. Burger, B.F. Lang, Mitochondrial evolution, Science 283 (1999) 1476-1481.

[17] J. Gubbens, P. Vader, J.M. Damen, M.C. O'Flaherty, M. Slijper, B. de Kruijff, A.I. de Kroon, Probing the membrane interface-interacting proteome using photoactivatable lipid cross-linkers, J. Proteome Res. 6 (2007) 1951-1962. 
[18] T. De Vrije, J. Tommassen, B. De Kruijff, Optimal posttranslational translocation of the precursor of PhoE protein across Escherichia coli membrane vesicles requires both ATP and the protonmotive force, Biochim. Biophys. Acta 900 (1987) 63-72.

[19] V. Reisinger, L.A. Eichacker, Analysis of membrane protein complexes by blue native PAGE, Proteomics 6 (Suppl. 2) (2006) 6-15.

[20] G. Candiano, M. Bruschi, L. Musante, L. Santucci, G.M. Ghiggeri, B. Carnemolla, P. Orecchia, L. Zardi, P.G. Righetti, Blue silver: a very sensitive colloidal Coomassie G-250 staining for proteome analysis, Electrophoresis 25 (2004) 1327-1333.

[21] A. Keller, A.I. Nesvizhskii, E. Kolker, R. Aebersold, Empirical statistical model to estimate the accuracy of peptide identifications made by MS/MS and database search, Anal. Chem. 74 (2002) 5383-5392.

[22] A.I. Nesvizhskii, A. Keller, E. Kolker, R. Aebersold, A statistical model for identifying proteins by tandem mass spectrometry, Anal. Chem. 75 (2003) 4646-4658.

[23] S.F. Altschul, T.L. Madden, A.A. Schaffer, J. Zhang, Z. Zhang, W. Miller, D.J. Lipman, Gapped BLAST and PSI-BLAST: a new generation of protein database search programs, Nucleic Acids Res. 25 (1997) 3389-3402.

[24] A.A. Schaffer, L. Aravind, T.L. Madden, S. Shavirin, J.L. Spouge, Y.I. Wolf, E.V. Koonin, S.F. Altschul, Improving the accuracy of PSI-BLAST protein database searches with composition-based statistics and other refinements, Nucleic Acids Res. 29 (2001) 2994-3005.

[25] R. Pitt-Rivers, F.S. Impiombato, The binding of sodium dodecyl sulphate to various proteins, Biochem. J. 109 (1968) 825-830.

[26] T. Takagi, K. Tsujii, K. Shirahama, Binding isotherms of sodium dodecyl sulfate to protein polypeptides with special reference to SDS-polyacylamide gel electrophoresis, J. Biochem. 77 (1975) 939-947.

[27] S.E. Ong, M. Mann, Mass spectrometry-based proteomics turns quantitative, Nat. Chem. Biol. 1 (2005) 252-262.

[28] X. Grandier-Vazeille, K. Bathany, S. Chaignepain, N. Camougrand, S. Manon, J.M. Schmitter, Yeast mitochondrial dehydrogenases are associated in a supramolecular complex, Biochemistry 40 (2001) 9758-9769.
[29] A.H. Millar, S.A. Hill, C.J. Leaver, Plant mitochondrial 2-oxoglutarate dehydrogenase complex: purification and characterization in potato, Biochem. J. 343 (1999) 327-334.

[30] E. Maas, H. Bisswanger, Localization of the alpha-oxoacid dehydrogenase multienzyme complexes within the mitochondrion, FEBS Lett. 277 (1990) 189-190.

[31] T. Toyoda, T. Sekiguchi, A. Takenaka, Crystallization of eukaryotic E3, lipoamide dehydrogenase, from yeast, for exhibiting X-ray diffraction beyond $2.5 \mathrm{~A}$ resolution, and preliminary structure analysis, J. Biochem. 121 (1997) 1-4.

[32] S. Meier, W. Neupert, J.M. Herrmann, Proline residues of transmembrane domains determine the sorting of inner membrane proteins in mitochondria, J. Cell Biol. 170 (2005) 881-888.

[33] E.E. Rojo, B. Guiard, W. Neupert, R.A. Stuart, Sorting of D-lactate dehydrogenase to the inner membrane of mitochondria. Analysis of topogenic signal and energetic requirements, J. Biol. Chem. 273 (1998) 8040-8047.

[34] M.G. Claros, J. Perea, Y. Shu, F.A. Samatey, J.L. Popot, C. Jacq, Limitations to in vivo import of hydrophobic proteins into yeast mitochondria. The case of a cytoplasmically synthesized apocytochrome b, Eur. J. Biochem. 228 (1995) 762-771.

[35] A. Krogh, B. Larsson, G. von Heijne, E.L. Sonnhammer, Predicting transmembrane protein topology with a hidden Markov model: application to complete genomes, J. Mol. Biol. 305 (2001) 567-580.

[36] D. Korbel, S. Wurth, M. Kaser, T. Langer, Membrane protein turnover by the m-AAA protease in mitochondria depends on the transmembrane domains of its subunits, EMBO Rep. 5 (2004) 698-703.

[37] J. Kyte, R.F. Doolittle, A simple method for displaying the hydropathic character of a protein, J. Mol. Biol. 157 (1982) 105-132.

[38] A. Tzagoloff, A. Barrientos, W. Neupert, J.M. Herrmann, Atp10p assists assembly of Atp6p into the F0 unit of the yeast mitochondrial ATPase, J. Biol. Chem. 279 (2004) 19775-19780.

[39] L. Jia, M.K. Dienhart, R.A. Stuart, Oxa1 directly interacts with Atp9 and mediates its assembly into the mitochondrial F1Fo-ATP synthase complex, Mol. Biol. Cell 18 (2007) 1897-1908. 